\title{
Hospital Medicine Update: High-Impact Literature from March 2018 to April 2019
}

\author{
Anna K Donovan, MD, MS ${ }^{1 *}$, Alfred Burger, MD, MSHCLD², \\ Christopher Moriates, MD³, Bradley A Sharpe, MD ${ }^{4}$, Carrie Herzke, MD, MBA 5
}

\begin{abstract}
${ }^{1}$ University of Pittsburgh School of Medicine, Pittsburg, Pennsylvania; ${ }^{2}$ Mount Sinai Beth Israel, Icahn School of Medicine at Mount Sinai, New York, New York; ${ }^{3}$ Dell Medical School at the University of Texas at Austin, Austin, Texas; ${ }^{4}$ University of California San Francisco Medical Center, San Francisco, California; Johns Hopkins Hospital, Baltimore, Maryland.
\end{abstract}

To assist busy hospital medicine clinicians, we summarized 10 impactful articles from last year. The authors reviewed articles published between March 2018-April 2019 for the Hospital Medicine Updates at the Society of Hospital Medicine and the Society of General Internal Medicine Annual Meetings. The authors voted to select 10 of 30 presented articles based on quality and clinical impact for this summary. The key findings include: (1) Vancomycin or fidaxomicin are the first-line treatment for initial Clostridioides difficile infection; (2) Unnecessary supplemental oxygen is linked to increased mortality; aim for a target oxygen saturation of $90 \%-94 \%$ in most hospitalized patients; (3) Stigmatizing language in medical records impacts physician trainees' attitudes and pain management practices; (4) Consider ablation for atrial fibrillation in patients with heart failure; (5) Patients with opioid use disorder should be offered buprenorphine or methadone therapy; (6) Apixaban is safe and may be preferable over warfarin in patients with atrial fibrillation and end-stage kidney disease; (7) It is probably safe to discontinue antimethicillin-resistant Staphylococcus aureus (MRSA) coverage in patients with hospital-acquired pneumonia who are improving and have negative cultures; (8) Selected patients with left-sided endocarditis (excluding MRSA) may switch from intravenous (IV) to oral antibiotics if they are clinically stable after 10 days; (9) Oral antibiotics may be equivalent to IV antibiotics in patients with joint and soft tissue infections; (10) A history-electrocardiogram-age-risk factors-troponin (HEART) score $\geq 4$ is a reliable threshold for determining the patients who are at risk for short-term major adverse cardiac events and may warrant further evaluation. Journal of Hospital Medicine 2019;14:XXX-XXX. () 2019 Society of Hospital Medicine

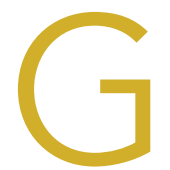

iven the breadth and depth of patients cared for by hospital medicine providers, it is challenging to remain current with the literature. The authors critically appraised the literature from March 2018 to April 2019 for high-quality studies relevant to hospital medicine. Articles were selected based on methodologic rigor and likelihood to impact clinical practice. Thirty articles were selected by the presenting authors for the Hospital Medicine Updates at the 2019 Society of Hospital Medicine (CH, CM) and Society of General Internal Medicine Annual Meetings $(B S, A B)$. After two sequential rounds of voting and group discussion to adjudicate voting discrepancies, the authors selected the 10 most impactful articles for this review. Each article is described below with the key points summarized in the Table.

\section{ESSENTIAL PUBLICATIONS}

Clinical Practice Guidelines for Clostridium difficile Infection in Adults and Children: 2017 Update by the Infectious Diseases Society of America (IDSA)

\footnotetext{
*Corresponding Author: Anna K. Donovan, MD, MS; Email: donovanak2@ upmc.edu; Telephone: 412-692-4888; Twitter: @AnnaKDonovan
}

Received: July 10, 2019; Revised: August 29, 2019; Accepted: August 29, 2019

(c) 2019 Society of Hospital Medicine DOI 10.12788/jhm.3321 and Society for Healthcare Epidemiology of America (SHEA). McDonald LC, et al. Clin Infect Dis. 2018;66(7):e1-e48. ${ }^{1}$

Background. In the United States, approximately 500,000 Clostridioides difficile infections (CDI) occur annually with 15,000-30,000 deaths. CDI has become a marker of hospital quality and has been placed under numerous "pay for performance" metrics. The Infectious Diseases Society of America/ Society of Healthcare Epidemiology of America updated their guidelines from 2010 regarding hospital surveillance, diagnostic testing, treatment, and infection precautions and control.

Findings. The panel included 14 multidisciplinary experts in epidemiology, diagnosis, infection control, and clinical management of adult and pediatric CDI. They used problem intervention comparison-outcome (PICO)-formatted, evidence-based questions. The selection of data and final recommendations were made in accordance with the GRADE criteria. A total of 35 recommendations were made.

Key clinical recommendations for hospitalists caring for adults: (1) Prescribe vancomycin or fidaxomicin over metronidazole for the initial treatment of CDI (strong recommendation, high quality of evidence); (2) Limit testing to the patients with unexplained new onset diarrhea, which is defined as $\geq 3$ unformed stools in 24 hours (weak recommendation, very low-quality evidence); (3) Avoid routine repeat testing within 
TABLE. Ten Articles, 10 Practical Implications.

\section{Practical Implications}

1. Vancomycin or fidaxomicin should be used for initial episodes of Clostridium difficile infections instead of metronidazole.

2. Unnecessary supplemental oxygen in the hospital may increase mortality. A target $\mathrm{SpO}_{2}$ of $90 \%-94 \%$ is reasonable for most hospitalized patients.

3. Words Matter! Stigmatizing language in the patient chart affects resident and student trainees' attitudes and prescribing practices.

4. Consider consulting electrophysiologists for patients with heart failure and atrial fibrillation, and look for more studies in the near future.

5. Patients with opioid use disorder should be offered buprenorphine or methadone therapy, especially after surviving a nonfatal overdose.

6. Consider use of standard dose ( $5 \mathrm{mg}$ twice a day) apixaban for patients with end-stage kidney disease and atrial fibrillation.

7. In patients with hospital-acquired pneumonia and who are clinically improving with negative cultures, discontinuing MRSA coverage is likely safe.

8. Selected patients with left-sided endocarditis (excluding MRSA) may be able to switch from intravenous to oral antibiotics if they are clinically stable after at least 10 days and can arrange for frequent outpatient follow-up.

9. Early data suggest that oral antibiotics are noninferior to intravenous antibiotics in patients with joint and soft tissue infections, but more data are needed before adopting oral treatment for all patients.

10. A HEART score of $>4$ can reliably be used as a cut off to stratify patients with chest pain in need of further evaluation versus those that can be sent home and who are at a low risk of short-term major cardiac events.

Abbreviation: MRSA; methicillin-resistant Staphylococcus aureus

seven days, and only test asymptomatic patients for epidemiologic reasons (strong recommendation, moderate-quality evidence); (4) Minimize the frequency and duration of highrisk antibiotic therapy and the number of antibiotic agents prescribed (strong recommendation, moderate quality of evidence); (5) Discontinue therapy with the inciting antibiotic agent as soon as possible (strong recommendation, moderate quality of evidence).

Caveats. As with the clinical application of any guidelines, individual case adjustments may be required.

Implications. Vancomycin or fidaxomicin should be used for the initial episode of CDI instead of metronidazole.

Mortality and Morbidity in Acutely III Adults Treated with Liberal versus Conservative Oxygen Therapy (IOTA): a Systematic Review and Meta-analysis. Chu DK, et al. Lancet. 2018;391(10131):1693-1705.

Background. Supplemental oxygen is often given to acutely ill hospitalized adults, even when they are not hypoxic or dyspneic. The safety and efficacy of this practice is unknown.

Findings. This systematic review and meta-analysis evaluated 25 randomized controlled trials enrolling 16,037 patients. Patients presented with several conditions, including sepsis, critical illness, stroke, myocardial infarction, and emergency surgery. The fraction of inspired oxygen in the liberal arms varied from $30 \%$ to $100 \%$. Most patients randomized to the conservative arm received no supplemental oxygen. Delivery of liberal oxygen to acutely ill adults was associated with increased in-hospital mortality (relative risk [RR]: $1.21 ; 95 \% \mathrm{Cl}$ : 1.03-1.43), 30-day mortality (RR: 1.14; 95\% Cl: 1.01-1.29), and 90-day mortality (RR: $1.10 ; 95 \% \mathrm{Cl}: 1.00-1.20$ ). The results were believed to be of high quality and were robust across multiple sensitivity analyses. It seemed that the mortality began to increase when supplemental oxygen raised the peripheral oxygen saturation $\left(\mathrm{SpO}_{2}\right)$ above a range of $94 \%-96 \%$.

Caveats. Heterogeneity was observed in the study settings and oxygen delivery. In addition, the cause for increased mortality could not be determined.

Implications. In hospitalized acutely ill adults, "liberal" supplemental oxygen was associated with increased in-hospital and longer-term mortality. The study authors postulated that this finding resulted from the direct toxic effects of oxygen or that oxygen delivery may "mask" illness and lead to delays in diagnosis and treatment. A subsequent clinical practice guideline recommends (1) a target $\mathrm{SpO}_{2}$ of less than $96 \%$ for patients receiving oxygen therapy; (2) a target $\mathrm{SpO}_{2}$ range of $90 \%-94 \%$ seems appropriate for most hospitalized adults. ${ }^{3}$

Do Words Matter? Stigmatizing Language and the Transmission of Bias in the Medical Record. P Goddu A, et al. J Gen Intern Med. 2018;33(5):68-91.4

Background. Previous work has shown that clinician bias affects health outcomes, often worsening health disparities. It is unknown whether clinicians' language in medical records biases other clinicians and whether this affects patients.

Findings. The investigators randomized medical students and residents in internal and emergency medicine at one academic medical center to review one of two vignettes in the format of notes on the same hypothetical patient with sickle cell disease (SCD) admitted with a pain crisis. One vignette contained stigmatizing language, and the other contained neutral language. The trainees exposed to the vignettes with stigmatizing language showed a more negative attitude toward the patient, as measured by a previously validated scale of attitudes toward patients with SCD (20.6 stigmatizing vs 25.6 neutral, with a total score range of 7-35 for the instrument; higher scores indicate more positive attitudes; $P<.001)$. Furthermore, the intensity of pain treatment was assessed in the resident group and was less aggressive when residents were exposed to stigmatizing language (5.56 stigmatizing vs 6.22 neutral on a scale of 2-7, with higher scores indicating more aggressive pain treatment; $P=.003$ ).

Cautions. This research was a single-center study of residents and medical students in two departments. Additionally, the study used vignettes on a hypothetical patient so trainees in the study group might have witnessed stronger stigmatizing language than what is typically observed in an actual patients' notes.

Implications. Stigmatizing language used in medical records possibly contributed to health disparities by negatively impacting other physicians' biases and prescribing practices toward patients with SCD at an academic medical center. Clinicians should avoid stigmatizing language in medical records. 
Catheter Ablation for Atrial Fibrillation with Heart Failure. Marrouche, NF et al. New Engl J Med. 2018;378:417-427. ${ }^{5}$

Background. Atrial fibrillation (AF) in patients with heart failure is associated with increased mortality and morbidity. Smallscale studies have suggested that ablation of AF may benefit patients with heart failure.

Findings. This multicenter trial included 398 patients with heart failure and symptomatic AF. Patients had New York Heart Association Class II-IV heart failure, an ejection fraction (EF) of $35 \%$ or less, and an internal cardiac defibrillator (ICD). Patients were randomized to either ablation or medical therapy. All enrolled patients either refused, failed, or showed poor tolerance to antiarrhythmic therapy for AF. The primary outcome was death from any cause or hospitalization for heart failure.

The composite endpoint occurred in $28.5 \%$ of the ablation group versus $44.6 \%$ of patients in the medical therapy group (hazard ratio [HR]: 0.62; 95\% Cl: 0.43-0.87). Fewer patients in the ablation group died (13\% vs $25 \%$; HR: 0.53 ; $95 \%$ Cl: $0.32-$ $0.86)$ or were hospitalized for heart failure (21\% vs $36 \%$; HR: $0.56 ; 95 \% \mathrm{Cl}: 0.37-0.83)$. The patients in the ablation group had higher EF increases above baseline and a greater proportion were in sinus rhythm at the 60-month follow-up visit.

Cautions. The trial was terminated early due to slow recruitment and lower than expected events. Over twice as many patients were lost to follow-up in the ablation group versus the medical therapy group, and by 60 months, AF recurred in $50 \%$ of patients who underwent ablation. The sample size was small, and the trial was unblinded.

Implications. Ablation should be considered for AF in patients with heart failure. Additional studies to evaluate ablation versus medical therapy for patients with heart failure and AF are underway.

Medication for Opioid Use Disorder after Nonfatal Opioid Overdose and Association with Mortality. Larochelle MR, et al. Ann Intern Med. 2018;169(3):137-145.6

Background. More than 70,000 Americans died of drug overdose in 2017; this number is higher than the deaths resulting from human immunodeficiency virus, car crash, or gun violence at their peaks. ${ }^{7}$ Methadone, buprenorphine, and naltrexone are approved by the Federal Drug Administration for the treatment of opioid use disorder (OUD). These medications increase treatment retention; methadone and buprenorphine have been associated with significant decreases in all-cause and overdose mortality. ${ }^{8}$ However, whether receipt of these medications following a nonfatal opioid overdose reduces mortality is unknown.

Findings. This retrospective cohort study included 17,568 opioid overdose survivors from the Massachusetts's Public Health Dataset between 2012 and 2014. Only three in 10 of these patients received any medications for OUD over 12 months following overdose. All-cause mortality was 4.7 deaths ( $95 \% \mathrm{Cl}$ : 4.4-5.0 deaths) per 100 person-years. The relative risk for all-cause mortality was $53 \%$ lower with methadone (adjust- ed hazard ratio [aHR]: $0.47 ; 95 \% \mathrm{Cl}: 0.32-0.71)$ and $37 \%$ lower with buprenorphine (aHR: $0.63 ; 95 \% \mathrm{Cl}: 0.46-0.87$ ).

Caveats. This cohort study may have missed confounders explaining why certain patients received medications for OUD. As a result, association cannot be interpreted as causation.

Implications. Methadone and buprenorphine are associated with a reduction in preventable deaths in patients with OUD who have survived an overdose. All patients with OUD should be considered for therapy.

Outcomes Associated with Apixaban Use in Patients with End-Stage Kidney Disease and Atrial Fibrillation in the United States. Siontis, KC, et al. Circulation. 2018;138:1519-1529.9

Background. Patients with end-stage kidney disease (ESKD) have poor outcomes when treated with warfarin for AF. These patients were excluded from clinical trials of direct oral anticoagulants. The goal of this study was to determine the outcomes of the use of apixaban in patients with ESKD and AF.

Findings. This retrospective cohort study included 25,523 Medicare patients with ESKD and AF on anticoagulants. A 3:1 propensity score match was performed between patients on warfarin and apixaban. Time without stroke/systemic embolism, bleeding (major, gastrointestinal, and intracranial), and death were assessed. A total of 2,351 patients were on apixaban, and 23,172 patients were on warfarin. No difference was observed in the risk of stroke/systemic embolism between apixaban and warfarin (HR 0.88; 95\% Cl: 0.69-1.12). Apixaban was associated with a lower risk of major bleeding (HR: 0.72; $95 \% \mathrm{Cl}: 0.59-0.87)$. Standard-dose apixaban (5 mg twice a day) was associated with lower risks of stroke/systemic embolism and death compared with reduced-dose apixaban $(2.5 \mathrm{mg}$ twice a day; $\mathrm{n}=1,317 ; \mathrm{HR}: 0.61 ; 95 \% \mathrm{Cl}: 0.37-0.98 ; P=.04$ for stroke/systemic embolism; HR: 0.64; 95\% Cl: 0.45-0.92; $P=.01$ for death) or warfarin (HR: 0.64; $95 \% \mathrm{Cl}: 0.42-0.97 ; P=.04$ for stroke/systemic embolism; HR: 0.63; 95\% Cl: 0.46-0.85; $P=.003$ for death).

Cautions. There may be unique patient factors that led providers to prescribe apixaban to patients with ESKD.

Implications. The use of standard-dose apixaban appears safe and potentially preferable in patients with ESKD and AF due to reductions in major bleeding, thromboembolism, and mortality risk compared with warfarin. Several additional studies are pending to evaluate the use and dose of apixaban in patients with ESKD and AF.

Outcomes Associated with De-escalating Therapy for Methicillin-Resistant Staphylococcus aureus in Culture-Negative Nosocomial Pneumonia. Cowley MC, et al. Chest. 2019;155(1):53-59. ${ }^{10}$

Background. Patients diagnosed with hospital-acquired pneumonia (HAP) are often treated empirically with broad-spectrum antibiotics. In many patients with HAP, cultures remain negative, and providers must decide if antibiotics can safely be narrowed. Specifically, the safety of deciding to "de-escalate" and discontinue the coverage for methicillin-resistant Staphy- 
lococcus aureus (MRSA) if cultures remain negative is unclear.

Findings. In this single-center retrospective cohort study, 279 patients who were (1) diagnosed with HAP and (2) had negative sputum cultures were enrolled. The patients in whom MRSA coverage was de-escalated by day four were compared with those with continued anti-MRSA coverage. No difference was observed between the two groups in terms of degree of illness or comorbidities. The patients who were de-escalated received five fewer days of anti-MRSA coverage than patients who were not. No difference was noted in the 28-day mortality between the two groups (de-escalation: $23 \%$ vs no de-escalation: $28 \%$; $95 \% \mathrm{Cl}$ : $-16.1 \%-6.5 \%)$. The incidence of acute kidney injury (AKI) was significantly lower in the de-escalation group (36\% vs $50 \%$; $95 \% \mathrm{Cl}$ : $-26.9-0.04$ ), and the overall length of stay was five days shorter in the de-escalation group $(95 \%$ Cl: 0.1-6.4 days).

Caveats. Given the retrospective nature, unmeasured confounders may have impacted the decision to de-escalate anti-MRSA coverage. The observed lower risk of AKI in the de-escalation group may be due to the simultaneous de-escalation of anti-Pseudomonas antibiotic agents in addition to the de-escalation of anti-MRSA coverage, as opposed to de-escalation of the anti-MRSA coverage alone.

Implications. De-escalation of anti-MRSA coverage in patients with HAP with negative cultures is associated with fewer antibiotic days, less AKI, and possibly shorter length of stay.

Partial Oral versus Intravenous Antibiotic Treatment for Endocarditis (POET). Iversen $\mathrm{K}$ et al. New Engl J Med. 2019;380(5):415-424. ${ }^{11}$

Background. Patients with left-sided infective endocarditis are typically treated with up to six weeks of intravenous (IV) antibiotics. The investigators studied the effectiveness and safety of switching to oral antibiotics after at least 10 days of IV therapy.

Findings. This randomized, multicenter, noninferiority trial at cardiac centers across Denmark included 400 adults with left-sided endocarditis who were clinically stable after at least 10 days of IV antibiotics. Half of the patients were randomized to continue IV therapy, whereas the other half was switched to oral antibiotics to complete the treatment course. Six months after therapy, no significant difference was observed between the two groups in terms of the primary composite outcomes, including all-cause mortality, unplanned cardiac surgery, embolic events, or relapse of bacteremia with the primary pathogen (IV-treated group: 12.1\%; orally treated group: $9.0 \%$ [between-group difference: $3.1 \% ; P=.40]$ ).

Caveats. A total of $20 \%$ of the screened population $(1,954$ adults) was randomized, and about $1 \%$ (5/400) of patients used injection drugs. None of the patients had MRSA. Patients in the oral group were assessed two to three times per week as outpatients, which may not be feasible in most settings.

Implications. Switching to oral antibiotics after at least 10 days of IV therapy appears to be safe and effective in selected patients with left-sided endocarditis. However, this study largely excluded patients with injection drug use and/or MRSA infections.
Oral versus Intravenous Antibiotics for Bone and Joint Infection (OVIVA). Li HK, et al. New Engl J Med. 2019;380(5):425-436. ${ }^{12}$

\section{Background}

Most complex orthopedic infections are treated with several weeks of IV antibiotics. This study sought to determine whether oral antibiotics are noninferior to IV antibiotics for bone and joint infections.

Findings. This randomized, multicenter, noninferiority, open-label trial of 1,054 adults with bone and joint infections in the United Kingdom included patients with prosthetic joints, other indwelling joint hardware, and native joint infections. Within seven days of antibiotic medication or within seven days of surgery (if performed), the patients received either IV or oral antibiotics for six weeks with a primary endpoint of treatment failure one year after the study randomization. The choice and duration of antibiotic treatment were determined by the involved infectious disease physician. A majority (77\%) of patients received greater than six weeks of therapy. Treatment failure was defined by clinical, microbiologic, or histologic criteria. Most enrolled patients were infected with Staphylococcus aureus, with $10 \%$ having methicillin-resistant $S$. aureus. Treatment failure was more frequent in the IV group than the oral group (14.6\% vs $13.2 \%)$, and these findings were consistent across all subgroups. More patients discontinued treatment in the IV group than the oral group.

Cautions. This study included a heterogenous population of patients with bone and joint infections, with or without hardware, and with different species of bacteria. Patients with bacteremia, endocarditis, or another indication for IV therapy were excluded. Limited injection drug use history was available for the enrolled patients. Most patients had lower limb infections. Thus, these findings are less applicable to vertebral osteomyelitis. Additionally, the study offered no comparison of specific antibiotics.

Implications. With appropriate oversight from infectious disease specialists, targeted oral therapy may be appropriate for the treatment of osteomyelitis. This shift in practice likely requires more study before broad implementation.

\section{Prognostic Accuracy of the HEART Score for Pre- diction of Major Adverse Cardiac Events in Patients Presenting with Chest Pain: A Systematic Review and Meta-analysis. Fernando S, et al. Acad Emerg Med. 2019;26(2):140-151.13}

Background. Chest pain accounts for over eight million emergency department (ED) visits yearly in the United States. Of those presenting with chest pain, 10\%-20\% will experience acute coronary syndrome (ACS) requiring further medical treatment. Given the fear of missing ACS, many low-risk patients are hospitalized. The American Heart Association has advocated using validated predictive scoring models to identify patients with chest pain who are at low risk for short-term major cardiovascular adverse event (MACE) for potential discharge without further testing. The authors evaluated the prognostic accuracy of higher risk scores to predict MACE in adult ED patients presenting with chest pain. 
Findings. This study was a systematic review and meta-analysis of 30 prospective and retrospective studies evaluating the history-electrocardiogram-age-risk factors-troponin (HEART) score through May 1, 2018. Meta-analysis compared the sensitivity, specificity, positive likelihood ratios, negative likelihood ratios, and diagnostic odds ratios of the HEART score and the Thrombolysis in Myocardial Infarction (TIMI) score when reported. An intermediate HEART score of 4-6 had a sensitivity of $95.9 \%$ and a specificity of $44.6 \%$. A high HEART score of $\geq 7$ had a sensitivity of $39.5 \%$ and a specificity of $95.0 \%$. Similarly, a high TIMI score of $\geq 6$ had a sensitivity of only $2.8 \%$ and a specificity of $99.6 \%$. The authors concluded that a HEART score of $\geq 4$ best identifies patients at risk of MACE who need greater consideration for additional testing.

\section{References}

1. McDonald LC, Gerding DN, Johnson S, et al. Clinical practice guidelines for clostridium difficile infection in adults and children: 2017 update by the infectious diseases society of America (IDSA) and Society for Healthcare Epidemiology of America (SHEA). Clin Infect Dis. 2018;66(7):e1-e48. https://doi. org/10.1093/cid/cix1085.

2. Chu DK, Kim LH, Young PJ, et al. Mortality and morbidity in acutely ill adults treated with liberal versus conservative oxygen therapy (IOTA): a systematic review and meta-analysis. Lancet. 2018;391(10131):1693-1705. https://doi. org/10.1016/S0140-6736(18)30479-3.

3. Siemieniuk RAC, Chu DK, Kim LH, et al. Oxygen therapy for acutely ill medical patients: a clinical practice guideline. BMJ. 2018;363:k4169. https://doi. org/https://doi.org/10.1136/bmj.k4169

4. A PG, O'Conor KJ, Lanzkron S, et al. Do words matter? Stigmatizing language and the transmission of bias in the medical record. J Gen Intern Med. 2018;33(5):685-691. https://doi.org/10.1007/s11606-017-4289-2.

5. Marrouche NF, Kheirkhahan M, Brachmann J. Catheter ablation for atrial fibrillation with heart failure. N Engl J Med. 2018;379(5):492. https://doi. org/10.1056/NEJMoa1707855.

6. Larochelle MR, Bernson D, Land T, et al. Medication for opioid use disorder after nonfatal opioid overdose and association with mortality: a cohort study. Ann Intern Med. 2018;169(3):137-145. https://doi.org/10.7326 /M17-3107.

7. Hedegaard HM, A; Warner, M. Drug Overdose Deaths in the United States,
Caveats. This meta-analysis failed to assess the potential adverse effects of false positive downstream testing. Additionally, no study compared the HEART score with the experienced clinician gestalt, which has often been equivalent to decision rules.

Implication. A HEART score $\geq 4$ risk stratifies ED patients with chest pain requiring further consideration for evaluation versus those that can be discharged with low risk for short-term MACE.

Disclosures: Dr. Burger owns stock in Portola Pharmaceuticals which was purchased on the open market and does not receive compensation for work. The company focuses primarily on drugs used in the treatment of thrombosis and hematological malignancies. All other authors have nothing to disclose.

1999-2017. 2018; https://www.cdc.gov/nchs/products/databriefs/db329. htm. Accessed March 07, 2019.

8. Medications for Opioid Use Disorder Save Lives. 2019; http://www.nationalacademies.org/hmd/Reports/2019/medications-for-opioid-use-disorder-save-lives.aspx. Accessed March 07, 2019.

9. Siontis KC, Zhang X, Eckard A, et al. Outcomes associated with apixaban use in patients with end-stage kidney disease and atrial fibrillation in the United States. Circulation. 2018;138(15):1519-1529. https://doi.org/10.1161/CIRCULATIONAHA.118.035418.

10. Cowley MC, Ritchie DJ, Hampton N, Kollef MH, Micek ST. Outcomes Associated With De-escalating Therapy for Methicillin-Resistant Staphylococcus aureus in Culture-Negative Nosocomial Pneumonia. Chest. 2019;155(1):5359. https://doi.org/10.1016/j.chest.2018.10.014

11. Iversen K, Ihlemann N, Gill SU, et al. Partial oral versus intravenous antibiotic treatment of endocarditis. N Engl J Med. 2019;380(5):415-424. https://doi. org/10.1056/NEJMoa1808312

12. Li HK, Rombach I, Zambellas R, et al. Oral versus Intravenous Antibiotics for Bone and Joint Infection. N Engl J Med. 2019;380(5):425-436. https://doi. org/10.1056/NEJMoa1710926

13. Fernando SM, Tran A, Cheng W, et al. Prognostic accuracy of the HEART score for prediction of major adverse cardiac events in patients presenting with chest pain: a systematic review and meta-analysis. Acad Emerg Med. 2019;26(2):140-151. https://doi.org/10.1111/acem.13649. 